\title{
Liposomal M-V-05: Formulation development and activity testing of a novel dihydrofolate reductase inhibitor for breast cancer therapy
}

\author{
BEE JEN TAN, KIAH SHEN QUEK, MAN-YI WONG, WAI KEUNG CHUI and GIGI N.C. CHIU \\ Department of Pharmacy, Faculty of Science, National University of Singapore \\ Block S4, 18 Science Drive 4, Singapore 117543
}

Received February 16, 2010; Accepted April 6, 2010

DOI: 10.3892/ijo_00000669

\begin{abstract}
In the management of metastatic breast cancer, fewer recognized therapeutic standards are available as compared to the early stages of the disease. Thus, it is pertinent to search for new, effective therapy to improve survival, tolerability and quality of life of patients. In this study, a liposomal formulation was developed for a novel dihydrofolate reductase (DHFR) inhibitor, M-V-05. Drug encapsulation into liposomes was achieved by the citrate-based, $\mathrm{pH}$ gradient loading technique, with a final drug-to-lipid weight ratio of $0.1: 1$. The liposome formulation exhibited a sustained release profile of the encapsulated drug that followed first order release kinetics. Liposomal M-V-05 was found to be more effective than the standard DHFR inhibitor, methotrexate, and its activity was comparable to liposomal doxorubicin, with $\mathrm{IC}_{50}$ values of 37 and $59 \mu \mathrm{M}$ achieved in MDA-MB-231 and JIMT-1 cells, respectively. Similar cytotoxicity was observed in primary patient samples of invasive ductal carcinoma of the breast. The combination of liposomal M$\mathrm{V}-05$ and liposomal doxorubicin in fixed molar ratio of 3:1 was additive in cytotoxicity, allowing the concentrations of liposomal doxorubicin and liposomal M-V-05 to be reduced by 62 and $46 \%$, respectively. Taken together, liposomal $\mathrm{M}-\mathrm{V}-05$ represents a promising agent and offers a potential new adjuvant therapy for breast cancer treatment.
\end{abstract}

\section{Introduction}

Breast cancer represents the most frequent cancer affecting women worldwide (1). While early detection and better treatment options might have improved the chances of surviving the disease, many patients still suffer from poor responses to chemotherapy and recurrences, especially those with estrogen

Correspondence to: Dr Gigi N.C. Chiu, Department of Pharmacy, Faculty of Science, National University of Singapore, Block S4, 02-09, 18 Science Drive 4, Singapore 117543

E-mail: phacncg@nus.edu.sg

Key words: dihydrofolate receptor, metastatic breast cancer, drug combination, synergism, liposomes receptor-negative (ER-) phenotype because of fewer therapeutic options available and an association with worse prognosis and higher mortality rates (2). Therefore, it is imperative to search for new agents that may have potential therapeutic activity against the ER- phenotype.

Among the molecular targets, dihydrofolate reductase (DHFR) which plays a crucial role in maintaining sufficient intracellular levels of tetrahydrofolate by recycling dihydrofolate has been exploited in the development of anti-cancer agents, as inhibition of this enzyme would lead to a decrease in cellular purines and thymidylate, and subsequently to a halt in DNA synthesis and cell division (3). Methotrexate is the first DHFR inhibitor developed with a long history of use in cancer chemotherapy. The recently approved multi-targeted anti-folate, pemetrexed, inhibits DHFR as one of its target enzymes, and exhibits activity against a broad spectrum of cancer types, including breast cancer (4-6). As normal cells also utilize DHFR for proliferation, those that are rapidly dividing would be affected by DFHR inhibition, and for instance, affected cells in bone marrow and in gastrointestinal tract lining would give rise to side-effects such as myelosuppression and mucositis (6-8). While supplementing with vitamins and folic acid could reduce treatment-related toxicities associated with the use of anti-folates (8), a drug delivery system that is capable of preferential delivery to tumors could be exploited in the formulation development of anti-folates.

Liposomes have been well established as drug carriers for a wide variety of anti-cancer agents, and a number of reviews have been published on the advancement of liposomal delivery as the enabling technology for anti-cancer drug delivery (9-11). One of the major advantages of liposomal delivery is the ability of liposomes to alter the pharmacokinetics and biodistribution of the encapsulated agent. Liposomes of sizes ranging from 50 to $150 \mathrm{~nm}$ are able to capitalize on the discontinuities in the tumor vascular endothelium and extravagate more readily as compared to normal healthy endothelium. Combining with an impaired lymphatic system in tumor tissues, liposomal systems allow increased preferential accumulation of the encapsulated agent in tumor site with a concomitant decrease in the extent and types of non-specific toxicities. The advantages of liposomal delivery have culminated in two approved liposomal formulations of doxorubicin in the treatment of metastatic breast cancer, with the dose- 
limiting cardiotoxicity much reduced and tolerability greatly improved (12-14).

Taking into consideration the promising results of DHFR inhibition and liposomal drug delivery in breast cancer therapy, the aim of the current study is to develop a liposome formulation for a novel DHFR inhibitor, M-V-05. The activity of liposomal M-V-05 was tested using two ER- cell lines, the metastatic MDA-MB-231 and the trastuzumabresistant, HER2-overexpressing JIMT-1, as well as primary patient samples of invasive ductal carcinoma (IDC) to establish its clinical utility. Furthermore, a fixed ratio combination of liposomal M-V-05 and liposomal doxorubicin was evaluated with the view of finding a potential new adjuvant therapy for breast cancer treatment.

\section{Materials and methods}

Materials. The drug, M-V-05, or 7,9-diamino-10-(3'-phenoxypropyloxy)-6,8,10-triazaspiro[4.5]deca-6,8-diene hydrochloride, was synthesized as previously described (15). 1,2-distearoylsn-glycero-3-phosphocholine (DSPC) was purchased from Avanti Polar Lipids (Alabaster, AL, USA). Trisodium citrate dihydrate and concentrated $\mathrm{H}_{2} \mathrm{SO}_{4}$ were obtained from Merck (Whitehouse Station, NJ, USA). DMEM/F12 medium was purchased from Invitrogen (Carlsbad, CA, USA). N-2-hydroxyethylpiperazine-N'-2-ethanesulfonic acid (HEPES) was obtained from AppliChem (Darmstadt, Germany). Sodium chloride salt was bought from Scan Asia Co., Ltd. Dimethyl sulfoxide (DMSO) and 3-(4,5-dimethylthiazolyl-2)-2,5diphenyltetrazolium bromide (MTT) were purchased from MP Biomedicals Inc. (Solon, OH, USA). Sephadex G-50 beads, octyl-ß-D-glucopyranoside (OGP), cholesterol (Chol) and all remaining chemicals were purchased from SigmaAldrich (St. Louis, MO, USA)

Preparation of liposomes. The liposomes were prepared by the thin film hydration method followed by extrusion as previously described (16). For M-V-05, the formulation was DSPC/Chol $(55: 45 \mathrm{~mol} / \mathrm{mol})$, and for doxorubicin, the formulation was DSPC/Chol/DSPE-PEG2000 (56:39:5 mol/ mol). Briefly, lipids were dissolved in chloroform and dried under a stream of nitrogen gas. The resulting lipid film was placed under high vacuum for a minimum of $2 \mathrm{~h}$. The dried lipid film was hydrated with sodium citrate $300 \mathrm{mM}(\mathrm{pH}$ adjusted to 4.0 ) at $65^{\circ} \mathrm{C}$ for $1-1.5 \mathrm{~h}$. The lipid preparation was subsequently extruded 10 times through stacked 0.1 - and $0.081 \mu \mathrm{m}$ polycarbonate filters at $65^{\circ} \mathrm{C}$ with a water-jacketed extruder apparatus (Lipex Biomembranes Inc.). Liposomal lipid concentrations were determined by the colorimetric phosphate assay described by Fiske and Subbarow (17). The mean diameter of the extruded liposomes was determined by Zetasizer 3000HSA particle sizer (Malvern).

Generation of transmembrane $\mathrm{pH}$ gradient and drug loading into liposomes. Extruded liposomes were passed down a Sephadex G-50 column pre-equilibrated with $20 \mathrm{mM}$ HEPES/150 mM NaCl buffer (HBS; pH 7.5) to generate the transmembrane $\mathrm{pH}$ gradient. The liposome lipid concentration was adjusted to $5 \mathrm{mg} / \mathrm{ml}$ and, subsequently, M-V-05 was added to achieve a drug-to-lipid weight ratio (D/L) of $0.1: 1$ and $0.2: 1$. For doxorubicin, the $\mathrm{D} / \mathrm{L}$ ratio for loading was 0.125:1 (w/w). The mixture was incubated at $65^{\circ} \mathrm{C}$. At specific time points, $100 \mu \mathrm{l}$ aliquots of the mixture were loaded onto 1-ml Sephadex G-50 spin columns to separate the encapsulated drug from the unencapsulated drug. Separation was done by centrifuging the $1 \mathrm{ml}$ spin columns at $680 \mathrm{x} \mathrm{g}$ for $3 \mathrm{~min}$. To determine drug concentration in the eluted sample, an aliquot was taken and adjusted to $0.1 \mathrm{ml}$ with HBS followed by the addition of $10 \mu \mathrm{l}$ OGP $100 \mathrm{mM}$ and $890 \mu 1$ HBS for M-V-05 or $900 \mu 1$ Triton-X-100 1\% for doxorubicin. The mixture was subsequently placed in a water bath $\left(>90^{\circ} \mathrm{C}\right)$ for $1-2 \mathrm{~min}$. After the mixture was cooled to room temperature, absorbance was measured $(\lambda=254 \mathrm{~nm}$ for M-V-05 and $480 \mathrm{~nm}$ for doxorubicin), and the drug concentration in the eluted liposome fraction was determined from a standard curve made from known drug concentrations. Liposomal lipid content in the eluted sample was determined by the Fiske and Subbarow phosphate assay (17). The final D/L was computed from the drug and liposomal lipid concentrations determined above, and compared to the initial D/L used for drug loading to calculate the loading efficiency (expressed as \% loading).

In vitro drug release assay. The membrane dialysis method was used to characterize the release kinetics of M-V-05 from liposomes, under a 1:600 sink condition with PBS (pH 7.4) at $37^{\circ} \mathrm{C}$. Briefly, $0.1 \mathrm{ml}$ aliquots of $\mathrm{M}-\mathrm{V}-05$ loaded liposomal sample were diluted with $0.4 \mathrm{ml} \mathrm{PBS}$, and transferred into Slide-A-Lyzer mini dialysis units of molecular weight cutoff: 3,500 (Pierce, Rockford, IL, USA). The dialysis units were suspended in a beaker containing $300 \mathrm{ml}$ PBS, with temperature maintained at $37^{\circ} \mathrm{C}$. At specified time points, the dialysis units were taken out from the beaker, and were analyzed for drug and liposomal lipid concentrations. For each time point, one dialysis unit was used.

Cell culture. MDA-MB-231 cells were obtained from American Type Culture Collection, and JIMT-1 cells were generously provided by Dr Marcel Bally (British Columbia Cancer Research Center, Vancouver, Canada). MDA-MB231 cells were grown as adherent monolayer cultures in RPMI-1640 medium supplemented with $10 \%$ fetal bovine serum and $1 \% \mathrm{~L}$-glutamine. Cells were maintained at $37^{\circ} \mathrm{C}$ in humidified air with $5 \% \mathrm{CO}_{2}$ and were sub-cultured using $0.25 \%$ trypsin with $1 \mathrm{mM}$ ethylenediamine tetraacetic acid (EDTA). JIMT-1 cells were cultured and supplemented in a similar manner with DMEM/F12 as the medium. Primary cell samples from patients with invasive ductal carcinoma of the breast were provided by NUH-NUS Tissue Repository, Singapore, and were cultured in MEGM mammary epithelial cell complete medium supplemented with bovine pituitary extract (Clonetic, Basel, Switzerland). The samples were used within three passages.

Cell viability assay. To assess the viability of cells after drug treatment, the MTT (3-(4,5-dimethylthiazolyl-2)-2,5-diphenyltetrazolium bromide) assay was used. Cells were seeded in 96 -well microtiter plates at 5000 cells $/ 0.1 \mathrm{ml}$ (unless specified otherwise) diluted in respective media. After incubating overnight at $37^{\circ} \mathrm{C}$, the medium in the well was 
removed and replaced with fresh medium $(0.1 \mathrm{ml} /$ well $)$. Drugs were diluted serially in respective media, and were added to the wells $(0.1 \mathrm{ml} /$ well $)$. Control wells consisted of cells in their respective media without treatment. Triplicates were performed for each drug concentration in each experiment unless specified otherwise. After $72 \mathrm{~h}$ of incubation, the MTT stock solution $(5 \mathrm{mg} / \mathrm{ml}$ in phosphate buffered saline, $\mathrm{pH}$ 7.4) was diluted 1:4 with media, and $50 \mu 1$ were added to each well. Plates were incubated for $4 \mathrm{~h}$ in humidified air with $5 \% \mathrm{CO}_{2}$ at $37^{\circ} \mathrm{C}$. After the incubation, the supernatant was carefully aspirated from each well. DMSO $(150 \mu 1)$ was added to each well to dissolve the formazan crystals, and the plate was gently shaken for $30 \mathrm{~min}$. Absorbance at $570 \mathrm{~nm}$ was measured with microplate reader (Tecan Infinite $^{\mathrm{TM}}$ 200, Tecan Trading AG, Switzerland). Cell viability was calculated as follows: viability $=\left(\mathrm{Abs}_{\mathrm{T}}-\mathrm{Abs}_{\mathrm{B}}\right)$ / $\left(\mathrm{Absc}^{-} \mathrm{Abs}_{\mathrm{B}}\right) \times 100 \%$, where $\mathrm{Abs}_{\mathrm{T}}, \mathrm{Abs}_{\mathrm{C}}$ and $\mathrm{Abs}_{\mathrm{B}}$ represent the absorbance readings from the treated cells, the control (untreated) cells, and background, respectively.

Analyses of drug combination effects. For studies involving drug combination treatment, results obtained from the MTT assay were used to compute the Combination Index (C.I.) using the CalcuSyn software version 2.0 (Biosoft, Cambridge, UK), based on the C.I. equation described by Chou and Talalay (18):

$$
\text { C.I. }=\frac{(D)_{1}}{\left(D_{x}\right)_{1}}+\frac{(D)_{2}}{\left(D_{x}\right)_{2}}
$$

where (D) $)_{1}$ and (D) $)_{2}$ are the doses of drug 1 and drug 2, respectively, that inhibit $\mathrm{x} \%$ in the experiment when they are used in combination. $\left(\mathrm{D}_{\mathrm{x}}\right)_{1}$ and $\left(\mathrm{D}_{\mathrm{x}}\right)_{2}$ are the doses of drug 1 and drug 2, respectively, that inhibit $\mathrm{x} \%$ in the experiment when they are used alone. Synergy is defined by a C.I. value of $<0.9$; additivity is defined by a C.I. ranged from 0.9 to 1.1 ; and antagonism is defined by a C.I. value of $>1.1$.

DNA content analyses by propidium iodide staining. After drug treatment, cells were harvested and fixed in cold ethanol $(70 \% \mathrm{v} / \mathrm{v})$ with density adjusted to $1 \times 10^{6}$ cells $/ \mathrm{ml}$. Subsequently, cells were stained in propidium iodide (PI) buffer $(50 \mu \mathrm{g} / \mathrm{ml} \mathrm{PI}, 1 \mathrm{mg} / \mathrm{ml}$ RNase A and $0.1 \%$ Triton X-100 in PBS) at $37^{\circ} \mathrm{C}$ for $15 \mathrm{~min}$ as previously described (19). Stained cells were chilled on ice for $1 \mathrm{~h}$ before analyses with Beckman Coulter Epics Altra flow cytometer. For each sample, 10,000 events were collected.

Statistical analyses. All data values are reported as mean \pm standard error of the mean (SEM). Statistical differences were determined using one-way analysis of variance (ANOVA) with Scheffe test used for post-hoc multiple comparisons. P-values of $<0.05$ were considered to be statistically significant.

\section{Results}

Encapsulation of M-V-05 into liposomes via transmembrane $\mathrm{pH}$ gradient. The synthesis of $\mathrm{M}-\mathrm{V}-05$ and its activity against A549 cancer cell line have been described previously (15).<smiles>NC1=NC2(CCCC2)N(OCCCOc2ccccc2)C(N)=N1</smiles>

Figure 1. Chemical structure of M-V-05, a novel DHFR inhibitor.

A.
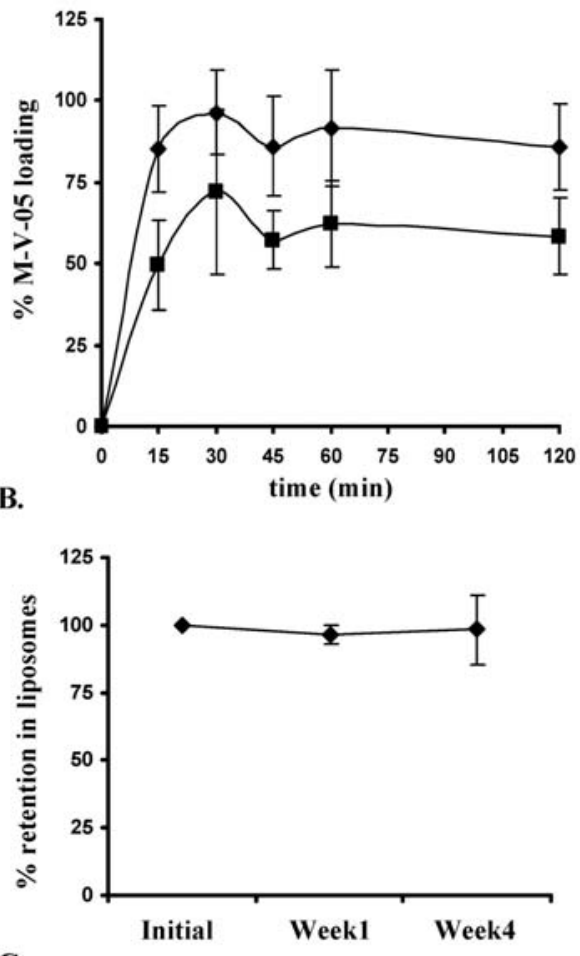

C.

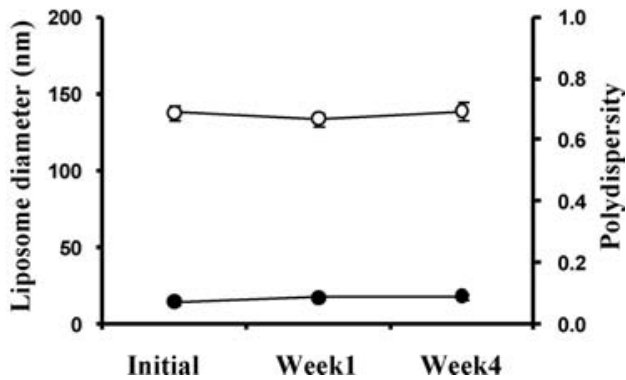

Figure 2. Time course of the loading of M-V-05 into liposomes via the transmembrane $\mathrm{pH}$ gradient method and the stability of the formulation. Two D/L weight ratios were investigated $(0.1: 1, * 0.2: 1, \mathbf{\square})$. At specific time points, \% M-V-05 loaded into liposomes was calculated as follows: \% loading $=($ final D/L) $/($ initial D/L) $\times 100 \%$ (panel A). Stability of liposomal M-V-05 (initial D/L $=0.1: 1$ ) at $4^{\circ} \mathrm{C}$ over a 4 -week period was monitored for $\%$ retention of drug (panel $\mathrm{B}$ ) and liposome diameter and polydispersity (panel C). Data points represent the mean of four independent experiments, with error bars indicating S.E.M.

With evidence that M-V-05 was able to reduce cancer cell viability, the first step in this study was to encapsulate $\mathrm{M}-\mathrm{V}-05$ into liposomes via the transmembrane $\mathrm{pH}$ gradient, as $\mathrm{M}-\mathrm{V}-05$ has a protonable amine group (Fig. 1). The lipid composition of the liposomes was DSPC/Chol 55:45 (mol/mol), and two $\mathrm{D} / \mathrm{L}$ weight ratios, 0.1:1 and 0.2:1 (w/w), were used for 
A.

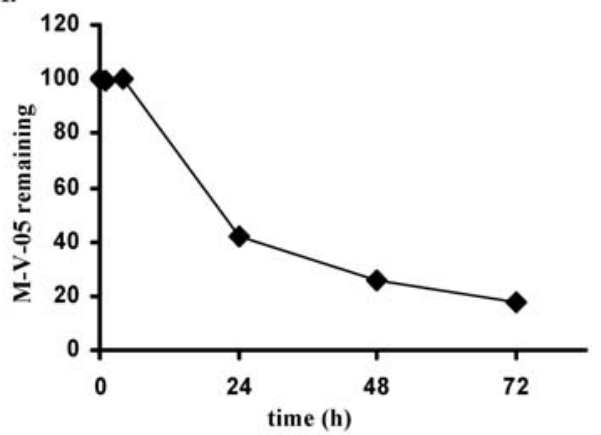

B.

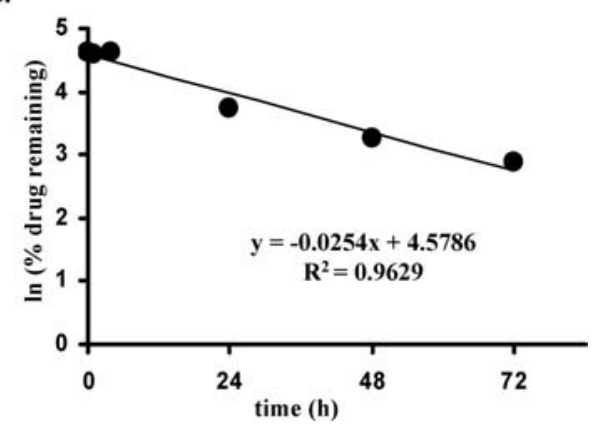

Figure 3. Release of M-V-05 from liposome at $37^{\circ} \mathrm{C}$ over $72 \mathrm{~h}$. Drug release was determined under a 1:600 v/v sink condition as described in Materials and methods. Panel A shows the percentage of $\mathrm{M}-\mathrm{V}-05$ remaining in liposomes over time, which was calculated using the following equation: \% remaining $=($ final $\mathrm{D} / \mathrm{L}) /($ initial $\mathrm{D} / \mathrm{L}) \times 100 \%$. Panel $\mathrm{B}$ shows the $\ln$ of the $\%$ of M-V-05 remaining in liposomes over time. Linear regression was used to find the line of best fit with the equation and $\mathrm{R}^{2}$ value included in the panel. The slope of the best fit line was used to calculate the time for $50 \%$ of $\mathrm{M}-\mathrm{V}-05$ released from the liposomes $\left(T_{1 / 2}\right)$.

loading. The loading temperature was set at $65^{\circ} \mathrm{C}$. These formulation attributes and loading conditions were chosen because they are commonly used in the production of liposomal drug products such as Doxil ${ }^{\circledR}$. As shown in Fig. 2A, a drug loading efficiency of $>90 \%$ could be achieved with 30 min of incubation when a $\mathrm{D} / \mathrm{L}$ ratio of $0.1: 1$ was used, and the $\mathrm{D} / \mathrm{L}$ ratio remained stable for the next $90 \mathrm{~min}$. When the $\mathrm{D} / \mathrm{L}$ ratio was increased to $0.2: 1$, the loading efficiency decreased to approximately $60 \%$. Thus, the optimal condition for loading $\mathrm{M}-\mathrm{V}-05$ into liposomes was a $\mathrm{D} / \mathrm{L}$ ratio of $0.1: 1$ with an incubation temperature of $65^{\circ} \mathrm{C}$ for at least $30 \mathrm{~min}$.

The stability of liposomal $\mathrm{M}-\mathrm{V}-05$ (initial $\mathrm{D} / \mathrm{L}=0.1: 1$ ) was monitored at $4{ }^{\circ} \mathrm{C}$ over a 4 -week period, and the assessment was based on the following parameters: 1) \% retention of drug over time, and 2) liposome diameter and polydispersity. As shown in Fig. 2B and C, $<10 \%$ of encapsulated M$\mathrm{V}-05$ was leaked from the liposomes upon storage at $4^{\circ} \mathrm{C}$, and no significant change in liposome size $(\mathrm{p}=0.767)$ and polydispersity ( $\mathrm{p}=0.450$ ) was observed during the monitoring period. These results suggest that liposomal M-V-05 is potentially a stable formulation for further development.

In vitro drug release profile of liposomal $M-V-05$. To characterize the release kinetics of $\mathrm{M}-\mathrm{V}-05$ from liposomes, the membrane dialysis method was used in which drug-loaded liposome samples were dialyzed against a large volume of PBS (pH 7.4) at $37^{\circ} \mathrm{C}$. In this study, a 1:600 sink condition was chosen, and the drug release profile was established over
A.

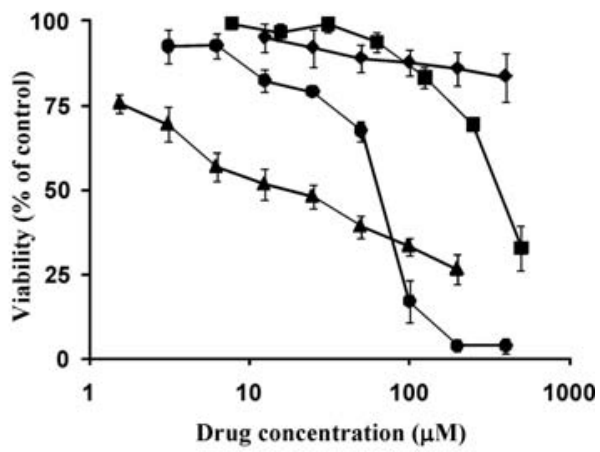

B.

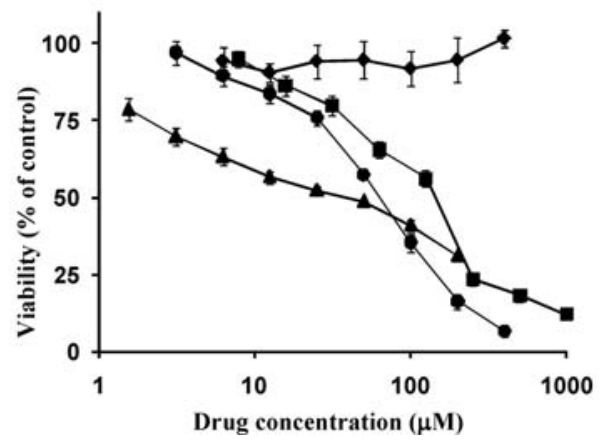

Figure 4. Liposomal M-V-05 reduced viability of two ER- breast cancer cell lines: MDA-MB-231 (A) and JIMT-1 (B). Drug treatment included

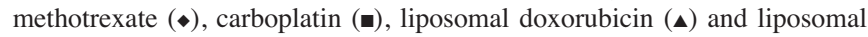
$\mathrm{M}-\mathrm{V}-05$ (•) for $72 \mathrm{~h}$. Cell viability was determined by the MTT assay, and was calculated as follows: viability $=\left(\mathrm{Abs}_{\mathrm{T}}-\mathrm{Abs}_{\mathrm{B}}\right) /\left(\mathrm{Abs}_{\mathrm{C}}-\mathrm{Abs}_{\mathrm{B}}\right) \mathrm{x} 100 \%$, where $\mathrm{Abs}_{\mathrm{T}}, \mathrm{Abs}_{\mathrm{C}}$ and $\mathrm{Abs}_{\mathrm{B}}$ represent the absorbance readings from the treated cells, the control (untreated) cells, and background, respectively. Each data point represents the mean of three independent experiments, with error bars indicating S.E.M

Table I. $\mathrm{EC}_{50}$ values $^{\mathrm{a}}(\mu \mathrm{M})$ of various drugs.

\begin{tabular}{lcc}
\hline & MDA-MB-231 & JIMT-1 \\
\hline Methotrexate & $>400$ & $>400$ \\
Carboplatin & $92(56-152)$ & $118(100-141)$ \\
Liposomal doxorubicin & $18(15-21)$ & $32(25-41)$ \\
Liposomal M-V-05 & $37(25-55)$ & $59(52-67)$ \\
\hline
\end{tabular}

${ }^{\mathrm{a}} \mathrm{EC}_{50}$ values were estimated using the CalcuSyn software version 2.0. Cell viability data obtained from the MTT assay were pooled from at least 3 independent sets and were used for the computation. Values given in parentheses represent the upper and lower 95\% confidence intervals of the $\mathrm{EC}_{50}$ estimations.

a time course of $72 \mathrm{~h}$. No significant change in the liposome diameter was observed when the samples were incubated under such conditions, indicating no aggregation of liposomes occurred (data not shown). As shown in Fig. 3A, release of M-V-05 from DSPC/Chol liposomes exhibited a sustained release profile. To further characterize the release kinetics, data presented in Fig. 3A were re-plotted with the natural logarithm $(\mathrm{ln})$ of \% $\mathrm{M}-\mathrm{V}-05$ remaining in liposomes against time. Fig. 3B presents the line of best fit to the data 
A.

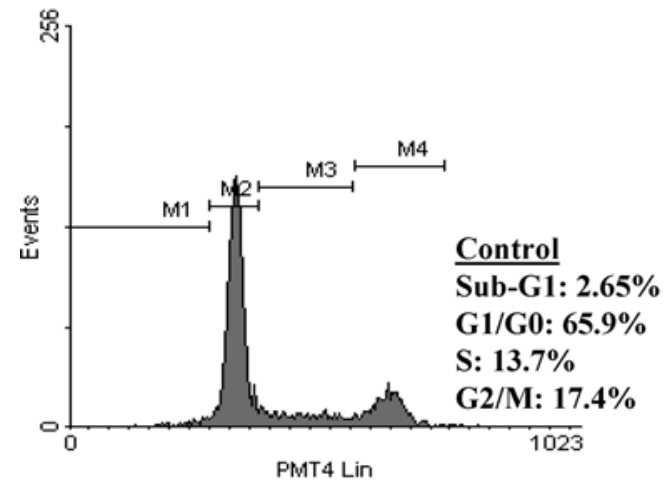

C.

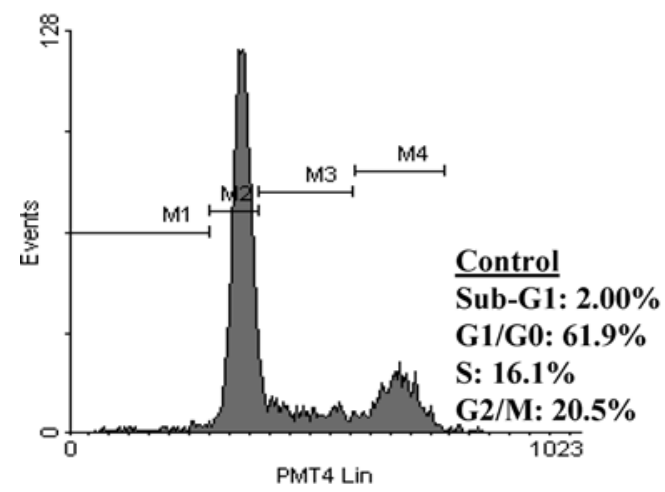

E.

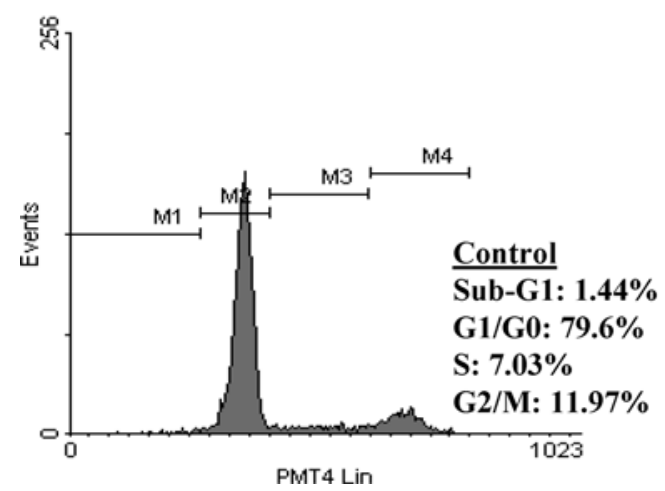

B.

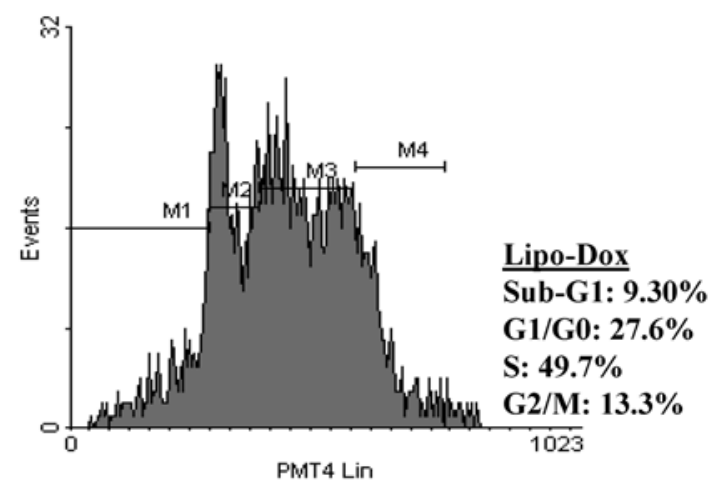

D.

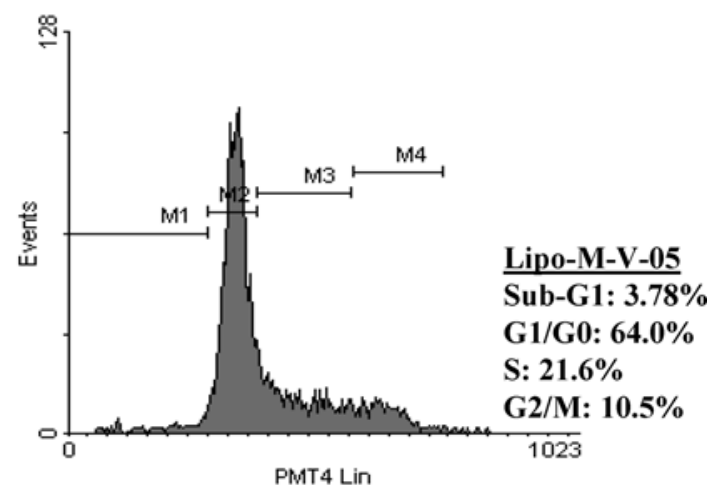

F.

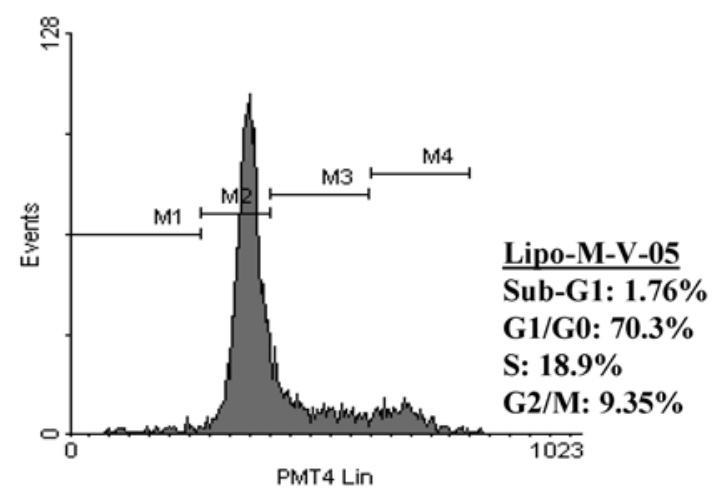

Figure 5. Cell cycle profiles of MDA-MB-231 cells with or without exposure to liposomal doxorubicin for $48 \mathrm{~h}$ (A, B) or to liposomal M-V-05 for 48 (C, D) and $72 \mathrm{~h}(\mathrm{E}, \mathrm{F})$. Cell samples were stained with propidium iodide as described in Methods before analyses by flow cytometry.

points after re-plotting, with $\mathrm{R}^{2}=0.9629$ and slope $=-0.0254$. This indicates that the release of $\mathrm{M}-\mathrm{V}-05$ followed first order kinetics, and the time for $50 \%$ of the encapsulated M-V-05 to be released $\left(T_{1 / 2}\right)$ from DSPC/Chol liposomes was $27 \mathrm{~h}$.

Ability of liposomal M-V-05 to reduce breast cancer cell viability. The activity of liposomal M-V-05 was tested in two ER- breast cancer cell lines, MDA-MB-231 and JIMT-1, and was compared to other drug treatments. Methotrexate was included as synthesis of M-V-05 was based on the pharmacophore of this drug. Carboplatin and liposomal doxorubicin were included as these two have been shown to be active in metastatic breast cancer $(20,21)$, and the latter is also a lipo- somal drug product that is approved in metastatic breast cancer therapy. Fig. 4 shows the cell viability data upon $72 \mathrm{~h}$ exposure to various drug treatments, and Table I summarizes the $\mathrm{EC}_{50}$ values. Compared to methotrexate and carboplatin, liposomal M-V-05 was more potent and effective against the two ER- breast cancer cell lines. The $\mathrm{EC}_{50}$ values of liposomal M-V-05 in MDA-MB-231 and JIMT-1 are 37 and $59 \mu \mathrm{M}$, respectively, which are in the same order as those of liposomal doxorubicin.

To further understand the cytotoxic effect mediated by liposomal M-V-05, DNA content analysis was performed on the treated cells using propidium iodide staining to examine any changes in the cell cycle status. As shown in Fig. 5, a 
A.

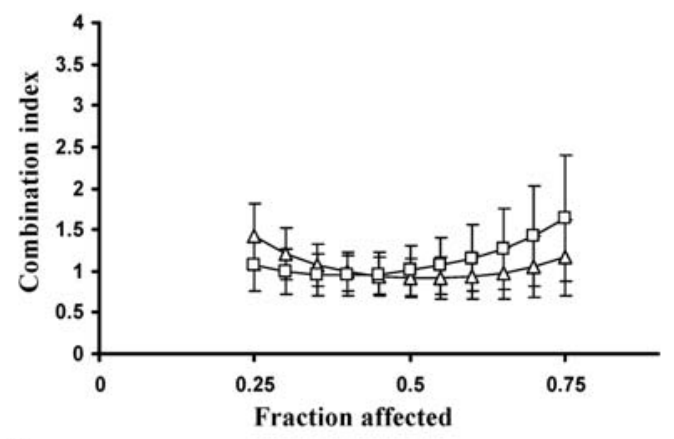

B.

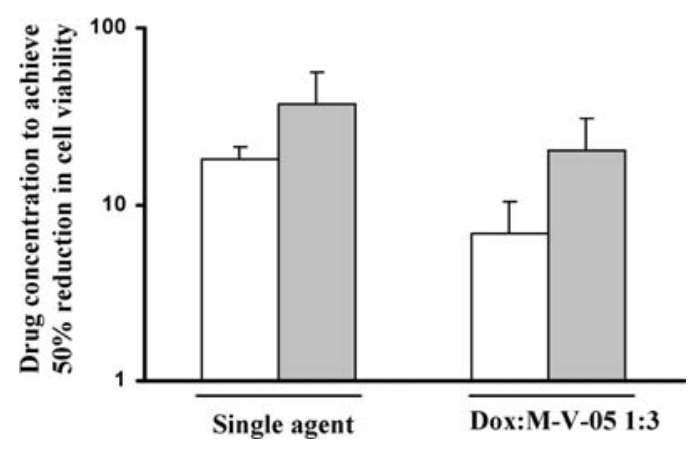

Figure 6. Effect of combining liposomal doxorubicin and liposomal M-V-05 in fixed molar ratios. MDA-MB-231 cells were treated for $72 \mathrm{~h}$ with liposomal doxorubicin and liposomal M-V-05 in 1:3 or 1:6 molar ratios, and viability was assessed by the MTT assay. Data collected were subsequently analyzed using the CalcuSyn software to determine the drug combination effects. Panel A: plot of combination index (C.I.) versus fa for liposomal doxorubicin and liposomal M-V-05 in 1:3 $(\triangle)$ or 1:6 ( $\square$ ) fixed ratios. Each data point represents the estimated C.I. $( \pm$ S.D. $)$ from three pooled sets of experiments. Panel B: dose reduction analyses for the combination of liposomal doxorubicin and liposomal M-V-05. The drug concentration to achieve 50\% reduction in cell viability was used for comparing the two liposomal drugs administered as single agents or in fixed ratio combination. Liposomal doxorubicin (white bars); liposomal M-V-05 (grey bars).

3.6-fold and a 2.7-fold increase in the proportion of cells in $\mathrm{S}$ phase was observed for a 48 -h exposure to liposomal doxorubicin and for a 72-h exposure to liposomal M-V-05, respectively. Concomitantly, the proportion of cells in G2/M phase was decreased after exposure to liposomal doxorubicin or to liposomal M-V-05.

Anti-cancer activity of the liposomal doxorubicin/liposomal $M-V-05$ combination. In cancer treatment, it is a rational approach to combine various anti-cancer drugs, in hope to lower drug doses and dose-dependent toxicities, yet at the same time, retain the therapeutic efficacy of the treatment if the drug combination exhibits non-antagonistic action. Thus, using MDA-MB-231 cells as the model, the two liposomal drug products, liposomal doxorubicin and liposomal M-V-05, were combined to further explore potential benefits. The median effect approach, described by Chou and Talalay (18), was used to assess whether fixed molar ratios of liposomal doxorubicin and liposomal M-V-05 would be synergistic (C.I.<0.9), additive (C.I.=0.9-1.1) or antagonistic (C.I.>1.1). Based on the $\mathrm{EC}_{50}$ values of the two liposomal drugs, 1:3 and 1:6 molar ratios of liposomal doxorubicin to liposomal $\mathrm{M}-\mathrm{V}$ 05 were selected for evaluation. As can be seen in Fig. 6A,
A.

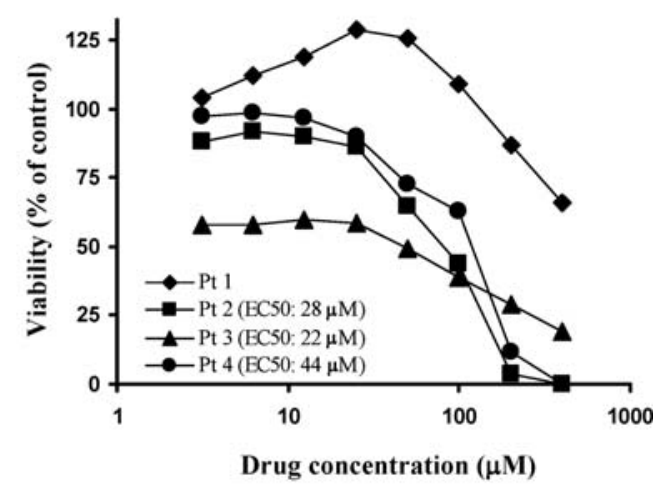

B.

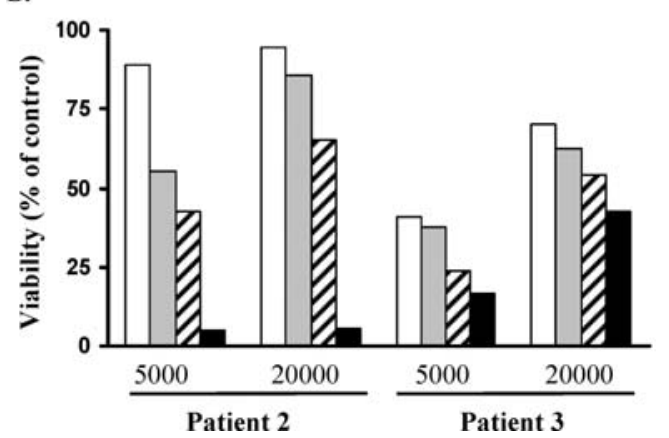

Figure 7. Liposomal M-V-05 reduced viability of primary breast cancer cells isolated from four patients diagnosed with invasive ductal breast carcinoma. Panel A: 10,000 cells were seeded into each well of a 96-well microplate, and were treated for $72 \mathrm{~h}$. $\mathrm{EC}_{50}$ values are given in parentheses for patients 2, 3 and 4. Panel B: for patient samples 2 and 3, two other cell seeding densities (5,000 and 20,000 per well) were tested with 25 (white), 50 (grey), 100 (hatched) and $200 \mu \mathrm{M}$ (black) liposomal M-V-05. Cell viability was determined by the MTT assay. Each data point represents a duplicate determination in one experiment.

the 1:3 ratio exhibited C.I. values of $0.9-1.1$ toward higher effect levels (fraction affected, fa $=0.5-0.75$ ), indicating additive effect from this liposomal drug combination. The 1:6 ratio exhibited a C.I. value of 1.0 at $\mathrm{fa}=0.5$, indicating additive effect; whereas slight antagonism (C.I.=1.6) was observed at a higher effect level (fa=0.75). Thus, the $1: 3$ ratio of liposomal doxorubicin/liposomal M-V-05 combination appears to be more promising. To further explore the possibility of dose reduction when the 1:3 ratio of liposomal doxorubicin and liposomal M-V-05 was used, the median drug concentrations of the two liposomal formulations used as single agents were compared to those obtained when the two formulations were combined. As shown in Fig. 6B, lower concentrations of liposomal doxorubicin and liposomal M-V-05 could be used to achieve $50 \%$ cell kill when the two liposomal drugs were combined. The concentrations of liposomal doxorubicin and liposomal M-V-05 were reduced by 62 and 46\%, respectively.

Activity of liposomal $M-V-05$ in primary breast patient samples. In addition to the two breast cancer cell lines, four patient breast cancer cell samples were obtained to establish the clinical utility of liposomal M-V-05. The primary cells were seeded at 10,000 cells/well and exposed to various concentrations of liposomal M-V-05 for $72 \mathrm{~h}$. Viability was determined by the MTT assay, and the results are presented 
in Fig. 7A. Of the four patient samples, three responded to the treatment of liposomal M-V-05, with $\mathrm{EC}_{50}$ values of 28 , 22 and $44 \mu \mathrm{M}$, respectively, which compare favorably to those obtained in the breast cancer cell lines. For patient 1 , cell viability was reduced to $63 \%$ at the highest concentration of liposomal M-V-05 used in the study. Two other initial cell seeding densities (5,000 and 20,000 cells/well) were explored in two patient samples to determine if liposomal M-V-05 could be effective over a range of cell numbers. As shown in Fig. 7B, liposomal M-V-05 was active even when the seeding density was increased from 5,000 to 20,000 cells/well, with $>50 \%$ reduction of viability achieved at $200 \mu \mathrm{M}$ liposomal M-V-05.

\section{Discussion}

In the management of metastatic breast cancer, fewer recognized therapeutic standards are available, especially after initial chemotherapy (22), as compared to early stage of the disease. Thus, it is pertinent to search for new, effective therapy to improve survival, tolerability and quality of life of patients. In this study, a liposomal formulation was developed for a novel DHFR inhibitor, M-V-05, and the activity of this formulation was tested using breast cancer cell lines as well as primary patient samples of IDC. Similar to other liposomal products such as Doxil and Myocet ${ }^{\circledR}$, the final drug-to-lipid weight ratio that could be achieved in the liposomal formulation of M-V-05 was 0.1:1. This formulation exhibited a sustained release profile (Fig. 3), which has the potential to avoid prolonged infusion time as opposed to the administration of anti-cancer drugs in their free form in order to maintain the minimum effective concentration.

We have demonstrated, using two breast cancer cell lines, that liposomal M-V-05 was more effective than the standard DHFR inhibitor, methotrexate and another commonly used drug, carboplatin, and its activity was in the same order as that of liposomal doxorubicin. When tested in the primary patient samples of IDC, liposomal M-V-05 exhibited similar cell killing activity as compared to its testing in the cell lines, with comparable $\mathrm{EC}_{50}$ values achieved in the patient samples. Furthermore, liposomal M-V-05 was effective over a wide range of cell numbers $(5,000-20,000)$, as shown in Fig. 7. Taken together, our results demonstrated the therapeutic potential of liposomal M-V-05. Further studies in animal models are warranted to understand the pharmacokinetic and toxicological profiles of the formulation as well as to investigate its therapeutic efficacy so as to establish its potential for full clinical utility.

Among the available conventional chemotherapeutic agents, anthracyclines such as doxorubicin are the most active against breast cancer, and they have been administered as the standard first-line therapy for the management of MBC. Furthermore, an anthracycline is often combined with other agents of non-overlapping toxicities and partial lack of crossresistance. Yet, it has been demonstrated that anthracyclinebased combination chemotherapy could be associated with increased treatment-related toxicity and worse quality of life in patients despite an improvement in response rates (23). Moreover, one major concern in the use of doxorubicin is its dose-limiting cardiotoxicity; other major side-effects include myelosuppression and alopecia. In overcoming the doselimiting cardiotoxicity, the use of drug delivery platforms such as liposomal delivery systems has been a success, resulting in two approved liposome formulations of doxorubicin. In the current study, we further demonstrated that the combination of liposomal M-V-05 with a pegylated liposome formulation of doxorubicin would yield additive cancer cell killing effect, with the potential of reducing the dose of liposomal doxorubicin needed for the same biological effect. As the pegylated liposome doxorubicin could give rise to palmar-plantar erythrodysesthesia (or hand-foot syndrome) with an overall incidence of $48 \%$ in MBC patients (24), it is thus beneficial to be able to reduce the dose of liposomal doxorubicin through the combinatorial use with liposomal M-V-05. In conclusion, liposomal M-V-05 is an effective therapy for breast cancer, and could be a novel adjuvant to liposomal doxorubicin therapy.

\section{Acknowledgements}

This research project is supported by Singapore Ministry of Education through the National University of Singapore Academic Research Fund Tier 1 (grant no. R-148-050-077101 and R-148-050-077-133).

\section{References}

1. Pecorelli S, Favalli G, Zigliani L and Odicino F: Cancer in women. Int J Gynaecol Obstet 82: 369-379, 2003.

2. Paone JF, Abeloff MD, Ettinger DS, Arnold EA and Baker RR: The correlation of estrogen and progesterone receptor levels with response to chemotherapy for advanced carcinoma of the breast. Surg Gynecol Obstet 152: 70-74, 1981.

3. Fleisher M: Antifolate analogs: mechanism of action, analytical methodology, and clinical efficacy. Ther Drug Monit 15: 521-526, 1993.

4. Adjei AA: Pemetrexed: a multitargeted antifolate agent with promising activity in solid tumors. Ann Oncol 11: 1335-1341, 2000.

5. Gomez HL, Santillana SL, Vallejos CS, et al: A phase II trial of pemetrexed in advanced breast cancer: clinical response and association with molecular target expression. Clin Cancer Res 12: 832-838, 2006

6. Hanauske AR, Chen V, Paoletti P and Niyikiza C: Pemetrexed disodium: a novel antifolate clinically active against multiple solid tumors. Oncologist 6: 363-373, 2001.

7. Cripps C, Burnell M, Jolivet J, et al: Phase II study of first-line LY231514 (multi-targeted antifolate) in patients with locally advanced or metastatic colorectal cancer: an NCIC Clinical Trials Group study. Ann Oncol 10: 1175-1179, 1999.

8. Martin M: Clinical experience with pemetrexed in breast cancer. Semin Oncol 33: S15-S18, 2006.

9. Hofheinz RD, Gnad-Vogt SU, Beyer U and Hochhaus A: Liposomal encapsulated anti-cancer drugs. Anticancer Drugs 16: 691-707, 2005.

10. Kozubek A, Gubernator J, Przeworska E and Stasiuk M: Liposomal drug delivery, a novel approach: PLARosomes. Acta Biochim Pol 47: 639-649, 2000.

11. Medina OP, Zhu Y and Kairemo K: Targeted liposomal drug delivery in cancer. Curr Pharm Des 10: 2981-2989, 2004.

12. Abraham SA, Waterhouse DN, Mayer LD, Cullis PR, Madden TD and Bally MB: The liposomal formulation of doxorubicin. Methods Enzymol 391: 71-97, 2005.

13. Chao TC, Wang WS, Yen CC, Chiou TJ, Liu JH and Chen PM: A dose-escalating pilot study of sterically stabilized, pegylated liposomal doxorubicin (Lipo-Dox) in patients with metastatic breast cancer. Cancer Invest 21: 837-847, 2003.

14. Cowens JW, Creaven PJ, Greco WR, et al: Initial clinical (phase I) trial of TLC D-99 (doxorubicin encapsulated in liposomes). Cancer Res 53: 2796-2802, 1993.

15. Ma X and Chui WK: Antifolate and antiproliferative activity of 6,8,10-triazaspiro[4.5]deca-6,8-dienes and 1,3,5-triazaspiro[5.5] undeca-1,3-dienes. Bioorg Med Chem 18: 737-743, 2010. 
16. Hope MJ, Nayar R, Mayer LD and Cullis PR: Reduction of liposome size and preparation of unilamellar vesicles by extrusion techniques. In: Liposome Technology. Vol 1. 2nd edition. CRC Press, Boca Raton, pp123-139, 1993.

17. Fiske $\mathrm{CH}$ and Subbarow Y: The colorimetric determination of phosphorus. J Biol Chem 66: 375-400, 1925.

18. Chou TC and Talalay P: Quantitative analysis of dose-effect relationships: the combined effects of multiple drugs or enzyme inhibitors. Adv Enzyme Regul 22: 27-55, 1984.

19. Warburton C, Dragowska WH, Gelmon K, et al: Treatment of HER-2/neu overexpressing breast cancer xenograft models with trastuzumab (herceptin) and gefitinib (ZD1839): drug combination effects on tumor growth, HER-2/neu and epidermal growth factor receptor expression, and viable hypoxic cell fraction. Clin Cancer Res 10: 2512-2524, 2004.

20. O'Brien ME, Talbot DC and Smith IE: Carboplatin in the treatment of advanced breast cancer: a phase II study using a pharmacokinetically guided dose schedule. J Clin Oncol 11: 2112-2117, 1993.
21. Salzberg M, Thurlimann B, Hasler U, et al: Pegylated liposomal doxorubicin (caelyx) in metastatic breast cancer: a communitybased observation study. Oncology 72: 147-151, 2007.

22. Metastatic breast cancer. Recommendations proposal from the European School of Oncology (ESO) - MBC Task Force. Breast 16: 9-10, 2007

23. Joensuu H, Holli K, Heikkinen M, et al: Combination chemotherapy versus single-agent therapy as first- and secondline treatment in metastatic breast cancer: a prospective randomized trial. J Clin Oncol 16: 3720-3730, 1998.

24. Leonard RC, Williams S, Tulpule A, Levine AM and Oliveros S: Improving the therapeutic index of anthracycline chemotherapy: focus on liposomal doxorubicin (myocet). Breast 18: 218-224, 2009. 